Jorge A. Sabato

\title{
El cambio tecnológico necesario y posible
}

¿Latinoamericanos, a las cosas mismas!

(Ortega y Gasset, Icremente modificado).

UNA PREGUNTA PREVIA

Este documento, que será breve y directo, se propone:

a) Hacer un balance del estado actual en América Latina de algunos de los aspectos fundamentales de lo que se ha dado en llamar la problemática ciencia-tecnología-desarrollo-dependencia.

b) Presentar para su discusión un mecanismo, el de la empresa de tecnologias, que se cree puede ayudar a hacer posible el cambio tecnológico necesario.

Hay, sin embargo, una pregunta previa: ¿se necesita tecnología? que plantea un cuestionamiento de fondo y que no puede soslayarse. La tecnología, que el optimismo panglossiano de los positivistas convirtió en fetiche, vuelve hoy a ser atacada duramente, como en su momento lo hicieron, con justicia, filósofos, anarquistas, románticos y surrealistas. Muchas voces la acusan de ser la causa fundamenta] de la crisis de nuestra civilización y otras tantas proclaman la urgencia de una vuelta al "estado natural". El hombre cosificado de Bretón, el hombre-engranaje de Ernesto Sabato, el hombre unidimensional de Marcusse, serian el resultado inexorable de la indiscriminada tecnologización de la cultura y su redención sólo podría lograrse por el rechazo total de una sociedad cientificista cuya máxima creación, la Máquina, habría transformado "al niño en obrero y al obrero en niño", como ha dicho K. Axelos parafraseando a Marx.

Se trata, por cierto, de una posición muy saludable, porque alerta sobre los graves peligros de idolatrar la tecnología, algo así como la entrega del alma colectiva a una suerte de diablo del siglo $\mathrm{xx}$, al tiempo que legitima potencias humanas tanto o más importantes que la racionalidad científica. No es posible, sin embargo, hacer ahora un análisis de su validez ontológica o de su coherencia epistemológica, y ni siquiera intentar un resumen de la profunda y dilatada polémica 
desatada a su alrededor. Se puede, en cambio, formular una observación muy simple, apenas una constatación, que lleva a una respuesta trivial pero suficiente en relación con los alcances de este trabajo: no hay, ni puede haber, producción sin tecnologia, sea aquella sim. ple y "natural" o sofisticada y "artificial", sea para satisfacer las necesidades básicas del hombre o para responder a sus demandas más superfluas. La tecnología, es, pues imprescindible, al par que es también inevitable, porque es "la reforma que el hombre impone a la naturaleza en vista de la satisfacción de sus necesidades", según la certera observación de Ortega y Gasset. Eventualmente, un hombre solitario podría sobrevivir algún tiempo sin tecnología, pero ello sería imposible para cualquier grupo humano. En consecuencia, en la medida que más seres humanos demanden más bienes y servicios, se necesitará más y más tecnología, porque ella es el motor del desarrollo de las fuerzas productivas; y que será por lo demás cada vez más sofisticada, sencillamente porque el hombre continúa profundizando el conocimiento de la naturaleza, y consecuentemente extendiendo su dominio y control, a través de más y más abstracción que es la causa primera de la malhadada sofisticación. De todo lo cual hay que alegrarse, porque sólo así será posible atender a las necesidades permanentes de los miles de millones de hombres que pueblan el planeta.

El problema no es entonces si se necesita tecnología sino el de saber qué tecnología se necesita, y la respuesta que se dé a esta pregunta definirá las relaciones que se establecen entre el hombre y esa inexorable herramienta de su realización. Así, la situación que se da hoy en la mayoría de los paises, tanto capitalistas como socialistas, es la consecuencia natural de que una determinada. Weltanschauung responde a esa cuestión no en términos de las necesidades fundamentales del ser humano sino de la sola racionalidad interna del sistema productivo. ¿Cómo puede extrañar entonces que la produccción ciega de tecnología o su adquisición indiscriminada produzcan graves daños en la trama misma de la sociedad?

Una respuesta diferente y que aparentemente permitiría al hombre liberarse de la esclavitud de su propia creación, podría ser la siguiente: la tecnologia que se necesita es aquella que ayude a proveer las necesidades básicas de la humanidad y a desarrollar en plenitud todas sus capacidades, empleando los recursos disponibles de manera que no conduzca a la explotación o sojuzgamiento del hombre ni a la destrucción irreversible de la naturaleza. Si se quiere que esta respuesta sea realizable, habrá que tener en cuenta que en la situación actual la mayor parte de la tecnologia que se utiliza, que se ofrece y que se busca, está lejos de satisfacerla y en consecuencia será imperativo desarrollar una capacidad autónoma para dirigir el 
proceso tecnológico, para manejar la tecnología a voluntad. Sólo mediante este manejo autónomo podrá una nación $\rightarrow 0$ grupo de naciones asociadas - comenzar a marchar en la dirección que eventualmente le permitirá disponer en cada caso de la tecnología más ajustada a sus propios objetivos, más respetuosa de su acervo cultural, más conveniente para sus propias necesidades y más adecuada a su constelación de recursos y factores. Así definiríamos el primero de los objetivos del cambio tecnológico necesario en América Latina.

Durante la última década se ha trabajado en América Latina con intensidad creciente en distintos aspectos de ciencia y tecnología; han ocurrido, y dejado de ocurrir, cosas importantes, por lo que un balance será útil para saber qué se ha hecho y dónde estamos. Antes, una observación importante: también se trabajó intensamente en las dos primeras décadas que siguieron a la Segunda Guerra Mundial, pero la actividad estuvo fundamentalmente dirigida a construir una infraestructura cientifico-técnica, a crear conciencia pública sobre la importancia de la ciencia y la impostergable necesidad de su desarrollo en nuestros países, campaña que culminó exitosamente con la creación de facultades de ciencia en numerosas universidades latinoamericanas, de institutos y centros de investigación y de consejos de investigaciones científicas y técnicas. Pero la tecnología estuvo casi totalmente ausente de ese esfuerzo porque se suponía que una vez puesta en marcha la máquina productora de ciencia, ésta fluiría de manera continua y se incorporaria sin mayores contratiempos a la realidad, que la estaba esperando ansiosamente. Es recién en los últimos diez años que la atención se centra en problemas como los siguientes: ¿Cuándo, por qué y cómo se crea demanda de ciencia en un momento histórico determinado? ¿Qué factores internos y externos determinan la oferta de la ciencia? ¿Cuáles son las relaciones entre ciencia y tecnología? ¿Es ésta simplemente "ciencia aplicada”? ¿Cómo circulan los flujos de la oferta y la demanda de tecnología por los distintos circuitos socioeconómicos? ¿A quiénes sirven los resultados de la inyestigación científico-tecnológica? ¿Cómo reaccionan los actores frente a las demandas externas? ¿Cómo y por qué se alienan la estructura productiva $y$ la infraestructura científico-técnica? ¿Qué papel le cabe al Estado? ¿Cuáles son las relaciones entre tecnología e inversión extranjera?, etc.

He aquí el balance: 
En lo académico, es decir, en el campo de los estudios e investigaciones de la problemática ciencia-tecnologia-desarrollo-dependencia, se han producido ideas originales, se han realizado agudos análisis teóricos, se han efectuado rigurosos estudios de campo y se han imaginado políticas y estrategias factibles de aplicación. El pensamiento latinoamericano así generado no está a la zaga del que se ubica "al más alto nivel internacional". Entre las realizaciones más significativas se cuentan (el orden de presentación no supone orden de méritos) :

a) Reconocimiento de la existencia de obstáculos estructurales al programa científico-técnico. Su estudio condujo a resultados importantes, tales como establecer la diferencia entre política científica explícita y politica científica implicita; comprender las causas del comportamiento corriente (hostilidad o indiferencia) de las élites gobernantes frente a la ciencia y la técnica, encontrar la racionalidad de la aparente contradicción entre el adelanto relativo de ciertas ramas de la ciencia (como la biología, por ejemplo) y el notorio atraso de otras (como la geología), descubrir las consecuencias del modelo por sustitución de importaciones en la incorporación de tecnología, tomar conciencia de la existencia de una nueva división internacional del trabajo centrada alrededor de la producción y consumo de tecnologia, etc.

b) Reconocimiento de la importancia de la tecnología como portadora de valores, y de los efectos socio-politico-culturales de la dependencia tecnológica.

c) Estudio en profundidad del comercio de tecnologia $\rightarrow$ a partir del reconocimiento de que ésta es una valiosa mercancía en el sistema productivo-, analizando sus mecanismos de operación, poniendo en evidencia las imperfecciones de su mercado, denunciando sus deformaciones más groseras, y penetrando en el sacralizado recinto de Ia propiedad industrial. Importancia de la "desagregación de tecnologia" o "apertura del paquete" en la importación de tecnología.

d) Reconocimiento del rol de las corporaciones transnacionales como productoras y comercializadoras de tecnología, lo que permitió dar transparencia a las relaciones inversión extranjera-tecnología. 
e) Estudios y proyectos para la planificación de la ciencia y la tecnología, sea a través de planes globales (indicativos y normativos) que intentan un ordenamiento desde la cúpula, sea mediante "regímenes de tecnologia" que la proponen en un proceso de "abajo hacia arriba".

f) Análisis crítico de la cooperación y ayuda científico-técnica multilateral y bilateral y de sus organismos y agencias de ejecución. Estudio de la transferencia de tecnología en sus diversos aspectos.

g) Análisis del rol del Estado y elaboración de instrumentos para la ejecución de políticas científicas y tecnológicas.

En lo político, es decir, en el campo de las acciones realizadas con el objeto de utilizar la ciencia y la tecnología para el logro de determinados objetivos, un aumento del nivel de conciencia en las élites gobernantes sobre la importancia del probiema (pese a que en muchos casos no ha trascendido de la mera retórica oficial) ha originado y fundamentado estrategias y políticas y se ha concretado en disposiciones juridicas y administrativas de importancia:

a) Formulación explicita de políticas científicas y tecnológicas y creación de órganos adecuados (ministerio y secretarias de estado) para su ejecución. Ejemplos destacados son las decisiones 84 y 85 del Pacto Andino y los dos planes de Ciencia y Técnica del Brasil.

b) Medidas para el análisis y control del flujo de tecnología importada (por ejemplo, la creación de los Registros de Tecnología) y para regular sus relaciones con la inversión de capitales extranjeros (Decisión 24 del Pacto Andino, ley de inversiones extranjeras de la Argentina, etc.).

c) Impulso de una nueva estrategia de cooperación y negociación en el nivel regional (OEA y SELA), subregional (Pacto Andino) e internacional (organismos de Naciones Unidas) y planteo de una nueva actitud frente a Estados Unidos (declaración de la conferencia CACTAL, posición en UNIDO y UNCTAD, etc.).

d) Aumento de la "tecnologización" de América Latina, medido por la mayor cantidad de personas que, en los distintos estratos de la sociedad, han adquirido capacidades científicas o habilidades técnicas. 
e) Aumento de la capacidad propia en ingeniería y consultoria y del intercambio científico-técnico entre los paises de la región y con el resto del mundo.

f) Algunos éxitos en la producción de tecnología (PEMEX de México, maquinaria agrícola en Argentina, máquinas herramientas en Brasil, etc.) y en la desagregación o "apertura del paquete tecnológico" (central nuclear de Atucha en Argentina, plan siderúrgico brasileño, petroquímica en el Pacto Andino, etc.).

g) Primeras exportaciones de tecnologia incorporada y desincorporada; medidas para su apoyo $y$ fomento (crédito preferencial, desgravaciones impositivas, tasas de cambio favorables).

\section{Debe}

En lo académico continúan sin resolver problemas muy importantes:

a) Muchos sectores siguen confundiendo ciencia y tecnología y creyendo que basta el desarrollo de aquélla para que ésta fluya normalmente. Esta confusión tiene graves consecuencias para ambas: para la ciencia, "la idea más difundida de lo que debiera ser, parece ser ésta: debiera ser empirica antes que teórica, regional antes que universal, aplicada antes que pura, natural antes que social, y en todo caso filosóficamente neutral...; estas cinco tesis... son nefastas" (Mario Bunge); para la tecnología, impide comprender los mecanismos de su producción y conduce inevitablemente a la mediocre perfomance de la mayoría de los centros e institutos de investigación industrial y aplicada existentes en la región. Esta misma confusión es la responsable de que la política científica y la política tecnológica se presenten como si fueran una sola y misma cosa.

b) La polémica cientificismo versus anticientificismo sigue planteada en términos tan estridentes como superficiales, en un nivel intelectual lamentable y con graves consecuencias prácticas. Por una parte, los alienados por la derecha siguen aferrados a un esquema de subordinación intelectual que es responsable en buena parte de la situación que padecemos; por otra, los alienados por la izquierda pueden llevarnos ( $\mathrm{y}$ en algunos países ya lo han hecho) a una situación que Hodara ha descrito certeramente: "Ia crítica ciega a la ciencia y a la tecnología como "palancas de dominio y explotación", la suspicacia excesiva hacia los centros del saber ubicados en los países industriales ( $y$ particularmente en los Estados Unidos) pue- 
de llevar -se reitera en el tiempo- a un bloqueo valorativo de ciertas disciplinas y acaso a un antintelectualismo generalizado que demolerá las bases fundamentales de la actividad que se declara fomentar".

Con parecida superficialidad se lleva a cabo el debate ciencia nacional versus ciencia internacional, que suele presentarse también como ciencia relevante, al servicio de los intereses concretos de la nación, versus ciencia alienada, ocupada de míticos y abstractos intereses de la humanidad. Se olvidan, por ignorancia a veces pero casi siempre por conveniencia, ciertos antecedentes fundamentales, como por ejemplo que en este siglo fueron los científicos nazis -en particular los Premios Nobel de Física P. Lenard y J. Stark - los que basaron la política científica de la Alemania de Hitler en los conceptos de "ciencia nacional" y "ciencia relevante" denunciando a los científicos judios (A. Einstein en particular) de ocuparse de problemas irrelevantes (como la naturaleza del espacio-tiempo o la dualidad onda-corpúsculo) en lugar de emplear su talento en el estudio de los problemas urgentes del pueblo alemán, que eran entonces la desocupación, la miseria y la humillación producida por el tratado de Versailles; también que en la década del 30 defendían la ciencia "nacional" Ios científicos de la "derecha" mientras que toda la "izquierda" intelectual se alienaba detrás de la bandera de la ciencia al servicio de la humanidad (exactamente a la inversa de lo que hoy ocurre); que la polémica condujo a situaciones dramáticas tanto en la URSS (caso Lysenko) como en Estados Uniclos (maccartismo), etcétera.

c) El problema central de la dependencia tecnológica ha sido denunciado con mucho vigor y estudiado con alguna amplitud pero lamentablemente no con la necesaria profundidad, por lo que son escasas y bastante precarias las propuestas formuladas para combatirla eficazmente. Lo mismo ocurre con la influencia de la tecnología en la distribución regresiva del ingreso y en el aumento del des: empleo y la marginalidad.

d) Los estudios sobre tecnología en alimentación, vivienda y salud son inferiores en calidad y cantidad a los realizados para el sector industrial, deformación que debe ser prontamente corregida si en verdad el foco de las preocupaciones es el conjunto de las necesidades básicas de las mayorías.

e) Se carece aún de una buena teoría sobre el rol del Estado como productor, propietario de unidades (industrias, bancos, seguros, comercios, etcétera) que son grandes consumidores de tecnología y que frecuentemente se comportan respecto a la ciencia y a la técnica 
en forma tanto o más regresiva que la del sector privado, desmintiendo así la creencia de que la nacionalización o estatización de una unidad productiva basta para terminar con su dependencia tecnológica.

f) No se ha avanzado lo suficiente en el conocimiento teórico de la cuantificación de los impactos de la inversión en ID sobre la estructura productiva como para permitir una planificación sustantiva.

En lo politico se está aún lejos de haber superado las contradicciones derivadas de la estructura dual de nuestras sociedades y los comportamientos tradicionales de las élites gobernantes, todo lo cual ha resultado en:

a) Las tecnologías destinadas a vivienda, alimentación y salud han recibido poca atención, mientras ha seguido creciendo la importación de tecnología destinada a atender la producción para el consumo de sectores privilegiados.

b) Las empresas de Estado no han sabido emplear su gran poder de compra para ayudar a la producción local de tecnología o a una mejor importación.

c) Las filiales de las corporaciones trasnacionales han continuado utilizando la importación de tecnologia para encubrir prácticas perniciosas.

d) No se han instrumentado incentivos fiscales adecuados para alentar la producción local de tecnología.

e) La infraestructura científico-técnica continúa desatendida y la inversión y el gasto están por debajo de las posibilidades reales en la mayoría de los países.

f) El brain drain ha continuado y en varios países ha aumentado fundamentalmente, por causa de la persecución politica. En cuanto al brain drain interno, buena parte de la infraestructura, y en especial las universidades, continúa aislada de la estructura productiva.

g) La demanda de tecnologia local sigue siendo muy débil, porque para la estructura productiva continúa siendo mejor negocio importar tecnología que producivla o adquirirla localmente. Conse- 
cuentemente, la oferta de tecnología local es débil e incapaz de organizarse adecuadamente.

h) La cooperación regional y subregional, esencial para poder alcanzar masa crítica y para poder atacar la multitud de problemas que deben ser resueltos, marcha muy lentamente, y particularmente con muy escasa capacidad de implementar los compromisos formales que se suscriben.

Este balance, que seguramente no es completo, permite sin embargo detectar el déficit mayor: somos fundamentalmente consumidores de tecnología, pero no productores; por lo tanto, espectadores y no autores, recipientes pasivos de lo que otros realizan en función de sus intereses y no de los nuestros, lo que nos lleva inexorablemente a adoptar esa Weltanschauung contra la que tanto se protesta y a la que se pretende derrotar con la mera retórica. Se llega así a una de dos posiciones igualmente nefastas: a la peor de las tecnolatrías, la del mimetismo o la copia o a la denuncia furibunda que esteriliza al no proponer alternativas viables. Para dominar lo que hoy nos domina hay que aprender a producir tecnologia, y producirla. Ello no garantiza de ninguna manera que podamos escapar de las trampas de una burda civilización cosificada y consumista, pero nos coloca sobre una plataforma sólida desde la cual intentar remontarnos. Sin ella seguiremos sumergidos y relegados, porque los que saben producir y producen seguirán en una situación de primacía, a favor de una necesidad objetiva de disponer de más y mejor tecnologia y de una neta ventaja comparativa para proveerla.

Hace ya 50 años Ortega y Gasset, con escaso éxito como se ve, incitó a los argentinos "a las cosas mismas". Hoy, abrumado por la retórica latinoamericana, fatigado por la literatura de denuncia y consciente de que el tiempo se nos va, me animo a convocar a los latinoamericanos para que en tecnología nos lancemos ya a las "cosas mis. mas", para lo cual me animo a formular una propuesta concreta.

UN MECANISMO POSHBLE

El mecanismo que aqui se propone ba sido descrito con todo detalle en un largo informe preparado para el PNUD en junio de 1975. En el convencimiento de que se trata de un mecanismo muy. idóneo para operar simultáneamente sobre dos áreas claves (producción de tecnologia y cooperación entre países de América Lati- 
na) he creido conveniente presentar a este Seminario una sintesis de sus principales caracteristicas, para que su discusión y posterior difusión ayuden a su más rápida puesta en operación.

Su fundamento es el siguiente: para lograr capacidad autónoma de manejo de la tecnología -que hemos definido como objetivo fundamental- es menester poder controlar el suministro de tecnología a la estructura productiva, de manera que llegue a ella la que más convenga, es decir, la tecnología que hemos definido más arriba como "aquella que ayude a proveer las necesidades básicas de la humanidad $y$ a desarrollar en plenitud sus capacidades, empleando los recursos disponibles de manera que no conduzca a la explotación o sojuzgamiento del hombre ni a la destrucción irreversible de la naturaleza"; con tal fin deberá actuarse sobre los mecanismos de producción y comercialización de tecnologia. Ello se puede lograr mediante la instalación y operación, en los más variados sectores de la estructura productiva, de empresas de tecnologia, unidades jurídico-administrativas que tienen por finalidad específica procesar conocimientos para producir y/o comercializar tecnología.

La empresa de tecnología es consecuencia natural de que en el sistema productivo la tecnología se comporta como una mercancía. Es decir, es objeto de transacciones comerciales y tiene precio: se compra y se vende, se permuta, se copia, se falsifica, se acumula, se roba, etc. Cumple así con la definición de Hunt y Schwartz: "Una mercancía es un objeto de utilidad producido para su intercambio en un mercado autónomo. En primer lugar, es un objeto útil; en segundo lugar, cuando se la lleva al mercado adquiere un status social definido, como un valor"; y con la de Boulding: "Una mercancía es algo que se intercambia y que por lo tanto tiene precio".

No significa esto que tecnología sea meramente nada más que mercancía y que por lo tanto se ignore o minimice su vasta dimensión cultural, en el sentido antropológico. Ocurre que si sólo se presta atención a esta dimensión es muy poco probable que se $1 \mathrm{ll}_{-}$ gue a comprender otros aspectos esenciales, ya que es sólo cuando se advierte su carácter de mercancía que se pueden formular preguntas claves que sólo entonces resultan obvias: ¿quién la produce?; ¿cómo se produce?; ¿cómo se vende?; ¿cómo se importa?; ¿cómo se financia?; etc. $\mathrm{X}$ estas preguntas llevan naturalmente a indagar si existe en el sistema productivo, una unidad especifica ocupada de este negocio particular; y si así ocurre, ¿cuál es?; ¿cómo está organizada?; ¿cómo funciona? Asi se llega a tomar conciencia de la existencia de una tal unidad, con la función especifica de procesar conocimiento para producir y comerciar tecnología, y a comprobar que en la estructura productiva se la encuentra por doquier, aunque muchas veces no se la descubra fácilmente. Lo que ocurre es que un 
velo semántico oculta su verdadera naturaleza; dicha unidad es una empresa y así debería llamarse, pero se la conoce generalmente con el nombre de laboratorio de investigación y desarrollo, o departamento de investigación y desarrollo o centro de investigación y dessarrollo o nombres similares en los que siempre figura la palabra investigación. Esta confusión lamentable resulta de que. las semejanzas formales entre una empresa de tecnología y un laboratorio de investigaciones al estado puro son grandes. En primer lugar, los elementos físicos son casi indistinguibles: edificios similares situados en paisajes parecidos, equipados con las mismas máquinas, instrumentos, aparatos, muebles y enseres, etc. La semejanza es aún mayor y más significativa en el personal: científicos y técnicos, con curricula similares, son dirigidos por hombres de altas calificaciones profesionales y académicas. También son semejantes el sistema de hábitos de trabajo, la división de tareas, la distribución de espacio y tiempo, la jerarquización profesional y hasta los lenguajes profesional y cotidiano de los que trabajan en ambas instituciones. Todas estas similitudes no son casuales, por cierto, sino la consecuencia de que la empresa organiza la producción de tecnología en base a las tareas de investigación que realizan sus "obreros y empleados", que son científicos y técnicos, colegas de los que trabajan en el laboratorio.

No debe extrañar entonces que tantas semejanzas oculten una diferencia esencial: mientras que en el laboratorio se busca el conocimiento por el conocimiento mismo (for the sake of it), en la empresa de tecnología se procesa el conocimiento (tanto el propio como el que se obtiene del stock universal) para producir la mercancía tecnología. Para el laboratorio, lo fundamental es encontrar la verdad; para la empresa, obtener un producto que sirva a las necesidades del cliente. En el laboratorio, el objetivo es la creación original; en la empresa, en cambio, es obtener respuesta a una demanda dada, respuesta que puede ser original o simplemente una adaptación o copia del resultado obtenido por otros. $\mathrm{E} 1$ conocimiento obtenido en el laboratorio tiene valor de uso; cuando se lo procesa en la fábrica adquiere valor de cambio. No es el cómo ni el con qué se realizan los trabajos los que los diferencia, ya que en ambos la herramienta fundamental (pero no la única en el caso de la empresa) es el método científico, sino el por qué y el para qué se los efectúa. Esta fundamental diferencia en los objetivos tiene efecto directo sobre la ética de los investigadores: mientras que el científico del laboratorio rige su comportamiento según dos principios cardinales: "No plagiarás" y "Publicarás los resultados de tus investigaciones", el que lo hace en una empresa de tecnología considera natural apropiarse indiscriminadamente de los resultados de los demás y ocultar 
cuidadosamente los suyos. Lo que para uno es sacrilegio, para el otro es una virtud que suele ser premiada generosamente.

Los conocimientos que emplea una empresa de tecnología pueden ser científicos o empíricos, propios o ajenos, originales o copiados, innovativos o adaptativos; prácticamente siempre el resultado del proceso es una mezcla adecuada de todas estas categorías; por eso es ocioso discutir si en una empresa de tecnología (ET) hay que hacer investigación básica o aplicada, moderna o antigua, para adaptar o para crear; hay que hacer aquello que sea más útil al proceso, que consiste en ensamblar, unir, conectar, entramar distintos conocimientos para producir así la tecnología deseada, que por eso mismo es un "paquete", siendo el objetivo de la ET "armar el paquete". Pero no sólo eso, porque además tiene que venderlo y para ello debe adicionar otros conocimientos, el conjunto de los cuales constituya una solución adecuada a las necesidades del cliente.

La ET es pues un centro de reunión y procesamiento de ideas, información y conocimiento provenientes de distintas fuentes: otras $\mathrm{ET}$, laboratorios nacionales y extranjeros, inventores independientes, universidades locales y extranjeras, empresas productivas, consultores, registros de patentes, literatura, espionaje industrial, etc. En una ET la capacidad de predecir las necesidades sociales o particulares, - convencer a un cliente para que los adquiera o utilice, justo con la competencia para aplicar conocimientos científicos y técnicas para resolver problemas concretos, son funciones que tienen más relevancia que la de producir brillantes soluciones científicas. La misión de una ET es proporcionar soluciones "adecuadas" a los problemas de sus clientes, y por tal se entiende que deberá elaborar tecnología que cumpla metas tales como: mayor producción, mejor productividad, más capacidad de desarrollo, etc. (en el caso de metas económicas) o combatir el desempleo, contribuir a combatir la marginalidad, proteger la naturaleza, etc. (en el caso de metas sociales) y que el mismo tiempo utilice racionalmente los recursos disponibles buscando menor costo de inversiones, mínima interferencia con las demás líneas de producción, mayor aprovechamiento de materias primas locales, etc.

La empresa de tecnología podría por cierto funcionar sin realizar investigación propia alguna, ya que en un principio le bastaría con usar el conocimiento que generan los laboratorios de investigación, y de hecho eso ocurre en numerosos casos. Pero la experiencia surgida del funcionamiento de muchas empresas ha demostrado que las más eficientes son aquellas que realizan una cierta cantidad de investigación propia, especialmente porque así pueden utilizar mejor el conocimiento generado por otros al par que reclutar mejores talentos para su "staff". Ocurre entonces que en esa tarea de investigación 
se suele producir conocimiento no aplicable inmediatamente, conocimiento puro o básico, como se le suele llamar. Tal cosa sucede naturalmente -por definición de investigación-y ese conocimiento puede ser de tan alta calidad como el mejor producido en laboratorios, al extremo de permitir a sus descubridores obtener recompensas académicas del más alto nivel, incluyendo el Premio Nobel, como ocurrió en 1932 (I. Langmuir, de la General Electric), en 1937 (Davisson, de la Bell) y en 1956 (Schockley, Brattain y Bardeen, también de la Bell). Pero a no engañarse: tales resultados y recompensas no son otra cosa que "externalidades" de la producción de la empresa de tecnología, ya que esta no existe para ganar premios académicos; si su personal los obtiene, tanto mejor, porque ello no sólo da prestigio a la empresa, sino que demuestra que tiene personal muy calificado $y$ que ha sabido organizarlo de modo tal de hacer posible la creación científica al nivel más alto; pero si la ET produjese sólo premios y recompensas no cumpliría su misión especifica y sin duda sería radicalmente reestructurada y posiblemente clausurada o quizá transformada en una fundación o universidad.

Por su parte, también los laboratorios de investigación científica suelen producir tecnologías, que son así "externalidades" de su función específica. Es natural que ello ocurra porque la tarea de investigación no tiene fronteras rígidas y por lo tanto muchos investigadores no se detienen en la simple obtención de un conocimiento, sino que se interesan y ocupan en su aplicación, realizando así tareas que pueden llegar a ser similares a las que se cumplen en una ET. Pero cuando estos desarrollos dejan de ser "externalidades" y se convierten en la razón de ser de la institución, el laboratorio se convierte en un "mission-oriented laboratory", que es nuevamente un nombre que oculta la realidad, porque lo cierto es que el laboratorio se ha transformado en empresa y así debería ser llamado, sobre todo para evitar que sus "operarios" no caigan en el error tan frecuente de creer que se espera de ellos que hagan ciencia, cuando su misión real es fabricar tecnología, produciéndose así una confusión de roles que suele producir pésimos resultados.

Con la ET la producción de tecnología se transforma de artesanal, es decir librada a la circunstancia fortuita de que a un inventor genial se le ocurriese una idea brillante o a un operario inteligente la modificación importante de una máquina en manufacturera, en la que resulta el fruto de un esfuerzo explícito y planeado, no ya de una persona sino de un grupo que puede llegar a ser muy numeroso. La consecuencia más importante de este cambio ha sido que la ET se ha constituido en el medio más apto de producción y comercialización de tecnología por lo que su desarrollo se ha acele- 
rado notablemente en las últimas décadas, como lo prueba el número, tamaño y variedad de ET que operan en los más diversos sectores de la economía de todos los países en todas partes del mundo. En particular, las corporaciones transnacionales se han mostrado muy eficientes en la organización de sus propias ET con las que han sido capaces de convertir a la tecnología en uno de los instrumentos más eficaces para la consolidación de su poder y riqueza. Todas ellas incluyen en su estructura una cierta capaciclad organizada de producir y comercializar tecnología, generalmente mediante una unidad responsable de su fabricación (y que debiera llamarse fábrica de tecnología y no departamento de investigación y desarrollo, como ocurre) para su propio consumo $y / 0$ para su venta, y una o más unidades ocupadas de su comercialización. Estas ET son cautivas en el sentido de que la tecnologia que elaboran se utiliza principalmente como insumo para la producción de las mercancías que constituyen el negocio fundamental de la corporación. Asi, por ejemplo, la tecnologia que manufactura la ET de una compaña como la General Electric está destinada, en primer lugar, a la fabricación de los diversos procluctos que GE lanza al mercado, como transformadores, turbinas, reactores nuclearcs, etc. En cambio hay otro tipo de ET -al que se podria llamar independiente- en la que la tecnología es el producto final, algo que es manufacturado para ser vendido como tal a otros que son los que lo emplearán como insumo de su propia producción. EI ejemplo más notorio es el de la Bell Telephone Laboratories que no produce teléfonos ni ningún otro artefacto sino exclusivamente tecnología para telecomunicaciones.

La organización de toda ET plantea los problemas clásicos de or. ganización de una empresa modema. En primer lugar los económico. financieros: ¿cuánto invertir? ¿cómo invertir? ¿cómo medir la rentabilidad de la inversión? ¿cómo presupuestar? ¿cuánto en bienes, cuánto en personal? ¿cuánto en gastos corrientes? ¿cómo evaluar imprevistos? ¿cómo afrontar los inevitables cambios de programa? Luego los inclustriales: ¿cómo instalar la fábrica? ¿cómo organizar la proclucción? ¿cómo medir la productividad? ¿cóno incentivar la producción? ¿cómo administrar el personal? Finalmente los problemas comerciales: ¿cómo evaluar el mercaclo? ¿cómo penetrarlo? ¿cómo hacer frente a la competencia? ¿cómo financiar läs ventas? ¿cómo exportar?

Las respuestas a la mayoria de estas preguntas son imprecisas y generalmente contradictorias, y hay sólidas razones para que asi ocurra. En primer lugar, la poca experiencia histórica en este tipo de producción, apenas umas pocas décadas agravada por el hecho de que se ha realizado y se realiza en sectores que son muy diferentes entre sí. Luego, la naturaleza especial del producto (tecnología) y de 
su insumo fundamental (ID), en la que la creatividad personal desempeña un papel esencial, por lo que la organización de un equipo de producción es tarea muy difícil ya que debe hacer compatible la eficiencia del conjunto con la ecuación individual de cada uno de sus componentes.

Asi como hay semejanzas y diferencias entre una ET y un laboratorio de investigaciones, también las hay con las empresas de consultoria y las empresas de ingenieria. Si en el primer caso -como ya hicimos notar- las semejanzas resultaban de la utilización por ambas del método científico y las diferencias del para qué lo utilizaban, en este segundo caso la situación se invierte: las semejanzas resultan del objetivo (ET, consultoras y empresas de ingenieria comercializan sus productos) y las diferencias de la metodología empleada para obtener el conocimiento, en la que la ET pone mayor énfasis en la producción de conocimiento mediante ID que lo que es tradicional en las otras empresas. Obviamente, hay diferencias de matices y por lo tanto suele ocurrir que unas se comporten como las otras y asi es dable reconocer la existencia de consultoras que son verdaderas ET y ET que funcionan como empresas de ingeniería. Pero el rango distintivo fundamental es que en la estrategia de largo plazo, toda ET debe incluir junto a la actividad de comercialización la actividad de producción, única forma de asegurar el desempeño cabal de su rol en la estructura productiva.

Con referencia a las ET, el cuadro de situación en América Latina es el siguiente:

a) Hay un cierto número de ET independientes en forma de organismos o instituciones del estado tales como los institutos nacionales de investigaciones industriales, los institutos de investigaciones agrícolas, las comisiones nacionales de energia atómica, los institutos del mar, del suelo, forestales, etc. Sin embargo, no sólo son pocos sino que también son débiles, a causa de: i) No se reconocen a sí mismos como ET y sufren por lo tanto los problemas derivados de la confusión de roles: ii) No incluyen funciones de comercialización de tecnologia, en particular en relación con la importación, por lo que quedan aislados de los problemas reales de utilización de la tecnología en sistema productivo; y iii) Tienen recursos escasos y mal administrados, en general por culpa cle las rigideces burocráticas que caracterizan a los gobiernos.

b) Hay muy pocas ET cautivas, localizadas generalmente en las grandes empresas de estado; las filiales de las corporaciones transnacionales no las necesitan porque su fuente principal de tecnologia 
está en las matrices, y las empresas de capital local no reciben estímulos suficientes para que les resulte conveniente instalarlas.

El balance es pues bastante negativo; hay pocas ET y funcionan con muy bajo rendimiento. Urge entonces fortalecer las que existen -sobre todo dándoles conciencia de su verdadero rol-e instalar muchas más, tanto independientes como cautivas, en los más diversos sectores de la economía, con lo que oportunamente se logrará disponer de la estructura de producción y comercialización de tecnología imprescindible para alcanzar la deseada autonomía.

Cuando se estudia la implantación de ET en los distintos sectores de la economía latinoamericana se Ilega a una conclusión muy interesante: existen sectores de importancia en los que sería no sólo posible sino conveniente implantar ET bi-, tri- o multinacionales (a las que llamaré EMT, empresas multinacionales de tecnología). Asi ocurre en energía eléctrica, siderurgia, petróleo, telecomunicaciones, aluminio, energía nuclear, etc. En efecto:

a) En todos los países, estos sectores son fuertes consumidores de tecnologia, y lo van a seguir siendo;

b) La mayor parte de las tecnologías que consumen son importadas, ya que la producción local es escasa o nula;

c) La mayoría de estas tecnologias importadas son iguales o similares para cada pais, simplemente porque las necesidades que tienen que satisfacer son similares;

d) En casi todos los paises de la región, estos sectores están en manos del estado y su empresa se realiza por empresas estatales o empresas mixtas con fuerte participación estatal;

e) También en casi todos los casos, los sectores no son competitivos entre sí, sino que atienden a sus propios mercados internos;

f) Cada una de esas empresas estatales necesita tecnología en un espectro muy amplio que cubre todas sus actividades; en consecuencia, es imposible que cada una pueda constituir una ET que sea capaz de atender con igual eficiencia todo ese rango.

Este conjunto de circunstancias hace factible la constitución de EMT, como se demuestra en el citado informe al PNUD en el que se proponen nueve ejemplos posibles en energía eléctrica, energía nuclear, ferrocarriles metropolitanos, matriceria, industria forestal, 
panificación, petróleo, plásticos y siderurgia. Que ello sería también conveniente resulta simplemente de apreciar que a través de EMT se podrá alcanzar el tamaño y el mercado mínimo sin los cuales la existencia de una ET sería muy precaria, se fortalecerá la capacidad de negociación para la importación de tecnologia, se racionalizará el empleo del recurso más escaso y costoso (el recurso humano) y se establecerán bases comunes para una producción autónoma de tecnología.

Hay un último aspecto de las EMrT que conviene subrayar: por definición, constituyen un instrumento apto para la cooperación entre paises; y aún más un instrumento que podría comenzar a funcionar muy rápidamente, por cuanto para constituir una EMT no se necesita pasar por acuerdos diplomáticos regionales o subregionales, ya que basta simplemente que se pongan de acuerdo dos países o dos empresas de esos dos países, para que la sociedad pueda funcionar. Posteriormente podrán incorporarse otras empresas de otros países, o asociarse entre ellas para constituir otra EMT, con lo que la cooperación seguirá extendiéndose. En todo caso no hay duda que con ellas se puede ir rápidamente "a las cosas mismas".

Con el mecanismo de las empresas de tecnología será posible:

a) Promover la producción de tecnología como actividad continua, diferenciada, específica y organizada, es decir, con identidad propia.

b) Sustentar la cooperación tecnológica alrededor de problemas concretos explícitamente definidos y de auténtico interés mutuo para todos los participantes, que serán justamente los que se asocien para constituir una dada empresa y que pueden pertenecer a distintos países de la región;

c) Privilegiar para su ejecución aquellos proyectos que cuenten con respaldo suficiente para su puesta en marcha y operación con el mínimo de dificultades institucionales y administrativas;

d) Disponer de un instrumento apto para ayudar a reducir la bre. cha existente entre los sectores moderno y tradicional vigentes actualmente en la totalidad de los países de la región;

e) Vincular una demanda concreta -económica o social- de innovación con la posibilidad de presentar una oferta concreta de conocimientos tecnológicos; 
f) Movilizar recursos humanos y materiales y'a existentes en la región para lograr su plena ocupación, contribuyendo asi a combatir tanto el brain drain externo como el interno;

g) Lograr la participación directa, tanto en el diseño como en la ejecución de un proyecto, de los "usuarios", de los "intermediarios o patrocinadores" y de los "productores" de tecnología;

h) Ayudar a luchar efectivamente contra la dependencia tecnolo. gica puesto que sólo la propia capacidad de producir tecnologia y de utilizarla con eficiencia puede permitir fundamentar una capacidad autónoma de decisión tecnológica.

Esta enumeración de beneficios no debe, sin embargo, llevar a engaño: el mecanismo propuesto puede llegar a ser condición necesaria para resolver el problema planteado y alcanzar el objetivo fijado, pero de ninguna manera será condición suficiente; para ello es imprescindible definir para qué se quiere la tecnología que se busca suministrar o, como hemos dicho más arriba, qué tecnología se necesita. Viceversa, no basta con definir esto último si luego no se sabe cómo realizarlo, como lo han probado ejemplos notorios de "liberación tecnológica" que no supieron pasar de la etapa declamatoria e incluso llevaron a aumentar la dependencia que se proponía combatir. 